\title{
Fixed Point Results for Multivalued Contractive Mappings Endowed with Graphic Structure
}

\author{
Tahair Rasham $\left(\mathbb{D},{ }^{1}\right.$ Abdullah Shoaib $\mathbb{D D}^{2},{ }^{2}$ Badriah A. S. Alamri, ${ }^{3}$ and Muhammad Arshad $\mathbb{D}^{1}$ \\ ${ }^{1}$ Department of Mathematics, International Islamic University, H-10, Islamabad 44000, Pakistan \\ ${ }^{2}$ Department of Mathematics and Statistics, Riphah International University, Islamabad 44000, Pakistan \\ ${ }^{3}$ Department of Mathematics, King Abdulaziz University, P.O. Box 80203, Jeddah 21589, Saudi Arabia
}

Correspondence should be addressed to Tahair Rasham; tahir_resham@yahoo.com

Received 9 August 2018; Accepted 27 November 2018; Published 19 December 2018

Academic Editor: Ali Jaballah

Copyright (C) 2018 Tahair Rasham et al. This is an open access article distributed under the Creative Commons Attribution License, which permits unrestricted use, distribution, and reproduction in any medium, provided the original work is properly cited.

\begin{abstract}
The aim of this paper is to introduce the notion of admissible multivalued mappings and to set up fixed point results for such mappings fulfilling generalized locally Ćirić type rational-contractive conditions on a closed ball in complete dislocated $b$-metric space. Example and application have been given to demonstrate the novelty of our results. Our results combine, extend, and infer several comparable results in the existing literature.
\end{abstract}

\section{Introduction and Preliminaries}

FP theory plays a fundamental role in functional analysis. Banach proved significant result for contraction mappings. After that, a huge number of FP theorems have been established by various authors and they made different generalizations of the Banach's result. Shoaib et al. [1], discussed the result related to $\alpha_{*}-\psi$-Ćirić type multivalued mappings on an intersection of a closed ball and a sequence with graph. Further FP results on a closed ball can be seen ([2-12]).

Boriceanu [13] proved FP results for multivalued generalized contraction on a set with two $b$-metrics. After this Aydi et al. [14] established FP theorem for set-valued quasi contraction in $b$-metric spaces. Nawab et al. [15] established the new idea of dislocated $b$-metric space as a conception of metric space and proved some common FP results for four mappings fulfilling the generalized weak contractive conditions in partially ordered dislocated $b$-metric space.

Nadler [16] initiated the study of FP theorems for the multivalued mappings (see also [17]). Several results on multivalued mappings have been observed (see [18-20]). Asl et al. [21] gave the idea of $\alpha_{*}-\psi$ contractive multifunctions and $\alpha_{*}$ admissible mapping and got some FP conclusions for these multifunctions (see also [22, 23]). In 1974, Ćirić [24] introduced quasi contraction. Reference [25] established some new common fixed points of generalized rational-contractive mappings in dislocated metric spaces with applications. In this paper, the concept of new Ćirić type rational multivalued contraction has been introduced. Now we prove new type of result for a different multivalued rational expression studied by Rasham et al. [6]. Common FP results for such contraction on a closed ball in complete dislocated $b$-metric space have been established. Example and application have been given. We give the following definitions and results which will be needed in the sequel.

Definition 1 (see [15]). Let $M$ be a nonempty set and let $d_{b}$ : $M \times M \longrightarrow[0, \infty)$ be a function, called a dislocated $b$-metric (or simply $d_{b}$-metric), if for any $g, q, \dot{z} \in M$ and $t \geq 1$ the following conditions hold:

(i) if $d_{b}(g, q)=0$, then $g=q$;

(ii) $d_{b}(g, q)=d_{b}(q, g)$;

(iii) $d_{b}(g, q) \leq t\left[d_{b}(g, \dot{z})+d_{b}(\dot{z}, q)\right]$.

The pair $\left(M, d_{b}\right)$ is called a dislocated $b$-metric space. It should be noted that the class of $d_{b}$ metric spaces is effectively larger than that of $d_{l}$ metric spaces, since $d_{b}$ is a $d_{l}$ metric when $t=1$.

It is clear that if $d_{b}(g, q)=0$, then from (i), $g=q$. But if $g=q, d_{b}(g, q)$ may not be 0 . For $g \in M$ and $\varepsilon>0, \overline{B(g, \varepsilon)}=$ 
$\left\{q \in M: d_{b}(g, q) \leq \varepsilon\right\}$ is a closed ball in $\left(M, d_{b}\right)$. We use D.B.M space instead of dislocated $b$-metric space.

Example 2. If $M=\mathbb{R}^{+} \cup\{0\}$, then $d_{b}(g, q)=(g+q)^{2}$ defines a D.B.M $d_{b}$ on $M$.

Definition 3 (see [15]). Let $\left(M, d_{b}\right)$ be a D.B.M space.

(i) A sequence $\left\{g_{n}\right\}$ in $\left(M, d_{b}\right)$ is called Cauchy sequence if, given $\varepsilon>0$, there corresponds $n_{0} \in N$ such that for all $n, m \geq n_{0}$ we have $d_{b}\left(g_{m}, g_{n}\right)<\varepsilon$ or $\lim _{n, m \rightarrow \infty} d_{b}\left(g_{n}, g_{m}\right)=$ 0 .

(ii) A sequence $\left\{g_{n}\right\}$ dislocated $b$-converges (for short $d_{b^{-}}$ converges) to $g$ if $\lim _{n \rightarrow \infty} d_{b}\left(g_{n}, g\right)=0$. In this case $g$ is called a $d_{b}$-limit of $\left\{g_{n}\right\}$.

(iii) $\left(M, d_{b}\right)$ is called complete if every Cauchy sequence in $M$ converges to a point $g \in M$ such that $d_{b}(g, g)=0$.

Definition 4. Let $\widehat{H}$ be a nonempty subset of D.B.M space $M$ and let $g \in M$. An element $q_{0} \in \widehat{H}$ is called a best approximation in $\widehat{H}$ if

$$
\begin{aligned}
& d_{b}(g, \widehat{H})=d_{b}\left(g, q_{0}\right) \\
& \text { where } d_{b}(g, \widehat{H})=\inf _{q \in \widehat{H}} d_{b}(g, q) .
\end{aligned}
$$

If each $g \in M$ has at least one best approximation in $\widehat{H}$, then $\widehat{H}$ is called a proximinal set.

We denote by $P(M)$ the set of all proximinal subsets of $M$. Let $\Psi$, where $t \geq 1$, denote the family of all nondecreasing functions $\psi:[0,+\infty) \longrightarrow[0,+\infty)$ such that $\sum_{k=1}^{+\infty} t^{k} \psi^{k}(u)<$ $+\infty$ and $t \psi(u)<u$ for all $u>0$, where $\psi^{k}$ is the $k^{\text {th }}$ iterate of $\psi$. Also $t^{n+1} \psi^{n+1}(u)=t^{n} t \psi\left(\psi^{n}(u)\right)<t^{n} \psi^{n}(u)$.

Definition 5 (see [26]). Let $B, A: M \longrightarrow P(M)$ be the closed valued multifunctions and $\beta: M \times M \longrightarrow[0,+\infty)$ be a function. We say that the pair $(B, A)$ is $\beta_{\star}$-admissible if for all $g, q \in M$

$$
\beta(g, q) \geq 1 \Longrightarrow \beta_{\star}(B g, A q) \geq 1,
$$

and $\beta_{\star}(A g, B q) \geq 1$,

where $\beta_{\star}(A g, B q)=\inf \{\beta(\bar{a}, b): \bar{a} \in A g, b \in B q\}$. When $B=A$, then we obtain the definition of $\alpha_{*}$-admissible mapping given in [21].

Definition 6. Let $\left(M, d_{b}\right)$ be a D.B.M space, $B: M \longrightarrow P(M)$ be multivalued mapping, and $\alpha: M \times M \longrightarrow[0,+\infty)$. Let $\bar{A} \subseteq M$, and we say that the $B$ is semi- $\alpha_{*}$-admissible on $\bar{A}$, whenever $\alpha(g, q) \geq 1$ implies that $\alpha_{*}(B g, B q) \geq 1$ for all $g, q \in$ $\bar{A}$, where $\alpha_{*}(B g, B q)=\inf \{\alpha(\bar{a}, b): \bar{a} \in B g, b \in B q\}$. If $\bar{A}=M$, then we say that the $B$ is $\alpha_{*}$-admissible on $M$.

Definition 7. The function $H_{d_{b}}: P(M) \times P(M) \rightarrow R^{+}$, defined by

$$
H_{d_{b}}(\bar{A}, B)=\max \left\{\sup _{\bar{a} \in \bar{A}} d_{b}(\bar{a}, B), \sup _{b \in B} d_{b}(\bar{A}, b)\right\},
$$

is called dislocated Hausdorff $b$-metric on $P(M)$.
Lemma 8. Let $\left(M, d_{b}\right)$ be a D.B.M space. Let $\left(P(M), H_{d_{b}}\right)$ is a dislocated Hausdorff $b$-metric space on $P(M)$. Then for all $\bar{A}, B \in P(M)$ and for each $\bar{a} \in \bar{A}$ there exists $b_{\bar{a}} \in B$ satisfying $d_{b}(\bar{a}, B)=d_{b}\left(\bar{a}, b_{\bar{a}}\right)$; then $H_{d_{b}}(\bar{A}, B) \geq d_{b}\left(\bar{a}, b_{\bar{a}}\right)$.

\section{Main Result}

Let $\left(M, d_{b}\right)$ be a D.B.M space, and let $g_{0} \in M$ and $B$ : $M \longrightarrow P(M)$ be the multifunctions on $M$. Then there exist $g_{1} \in B g_{0}$ such that $d_{b}\left(g_{0}, B g_{0}\right)=d_{b}\left(g_{0}, g_{1}\right)$. Let $g_{2} \in B g_{1}$ be such that $d_{b}\left(g_{1}, B g_{1}\right)=d_{b}\left(g_{1}, g_{2}\right)$. Continuing this process, we construct a sequence $g_{n}$ of points in $M$ such that $g_{n+1} \epsilon$ $B g_{n}, d_{b}\left(g_{n}, B g_{n}\right)=d_{b}\left(g_{n}, g_{n+1}\right)$. We denote this iterative sequence by $\left\{M B\left(g_{n}\right)\right\}$. We say that $\left\{M B\left(g_{n}\right)\right\}$ is a sequence in $M$ generated by $g_{0}$.

Theorem 9. Let $\left(M, d_{b}\right)$ be a complete D.B.M space, $\dot{r}>$ $0, g_{0} \in \overline{B_{d_{b}}\left(g_{0}, r\right)}$, and $B: M \longrightarrow P(M)$ be a semi- $\alpha_{*}-$ admissible multifunction on $\overline{B_{d_{b}}\left(g_{0}, \dot{r}\right)} ;\left\{M B\left(g_{n}\right)\right\}$ is a sequence in $M$ generated by $g_{0}, \alpha\left(g_{0}, g_{1}\right) \geq 1$. Assume that, for some $\psi \in \Psi$ and

$$
\begin{aligned}
& D_{b}(g, q)=\max \left\{d_{b}(g, q), \frac{d_{b}(g, B g) \cdot d_{b}(q, B q)}{\bar{a}+d_{b}(g, q)},\right. \\
& \left.d_{b}(g, B g), d_{b}(q, B q)\right\}
\end{aligned}
$$

where $\bar{a}>0$, the following hold:

$$
\begin{aligned}
& \alpha_{*}(B g, B q) H_{d_{b}}(B g, B q) \leq \psi\left(D_{b}(g, q)\right) \\
& \forall g, q \in \overline{B_{d_{b}}\left(g_{0}, \dot{r}\right)} \cap\left\{M B\left(g_{n}\right)\right\} \\
& \sum_{i=0}^{n} B^{i+1}\left\{\psi^{i}\left(d_{b}\left(g_{0}, g_{1}\right)\right)\right\} \leq \dot{r} \quad \forall n \in \mathbb{N} \cup\{0\} .
\end{aligned}
$$

Then, $\left\{M B\left(g_{n}\right)\right\}$ is a sequence in $\overline{B_{d_{b}}\left(g_{0}, r\right)}, \alpha\left(g_{n}, g_{n+1}\right) \geq 1$, and $\left\{M B\left(g_{n}\right)\right\} \longrightarrow g^{*} \in \overline{B_{d_{b}}\left(g_{0}, \dot{r}\right)}$. Also if $\alpha\left(g_{n}, g^{*}\right) \geq 1$ or $\alpha\left(g^{*}, g_{n}\right) \geq 1$, for all $n \in \mathbb{N} \cup\{0\}$ and the inequality (5) holds for all $g, q \in \overline{B_{d_{b}}\left(g_{0}, \dot{r}\right)} \cap\left\{M B\left(g_{n}\right)\right\} \cup\left\{g^{*}\right\}$, then $B$ has a common fixed point $g^{*}$ in $\overline{B_{d_{b}}\left(g_{0}, r\right)}$.

Proof. Consider a sequence $\left\{M B\left(g_{n}\right)\right\}$ generated by $g_{0}$. Then, we have $g_{n} \in B g_{n-1}$, and $d_{b}\left(g_{n-1}, B g_{n-1}\right)=d_{b}\left(g_{n-1}, g_{n}\right)$, for all $n \in \mathbb{N}$. By Lemma 8 , we have $d_{b}\left(g_{n}, g_{n+1}\right) \leq H_{d_{b}}\left(B g_{n-1}, B g_{n}\right)$ for all $n \in \mathbb{N}$. If $g_{0}=g_{1}$, then $g_{0}$ is a fixed point in $\overline{B_{d_{b}}\left(g_{0}, \dot{r}\right)}$ of $B$. Let $g_{0} \neq g_{1}$. From (6), we have

$$
d_{b}\left(g_{0}, g_{1}\right) \leq \sum_{i=0}^{n} \psi^{i}\left(d_{b}\left(g_{0}, g_{1}\right)\right) \leq \dot{r} .
$$

It follows that

$$
g_{1} \in \overline{B_{d_{b}}\left(g_{0}, \dot{r}\right)}
$$

If $g_{1}=g_{2}$, then $g_{1}$ is a fixed point in $\overline{B_{d_{b}}\left(g_{0}, \dot{r}\right)}$ of $B$. Let $g_{1} \neq g_{2}$. Since $\alpha\left(g_{0}, g_{1}\right) \geq 1$ and $B$ is semi- $\alpha_{*}-$ admissible 
multifunction on $\overline{B_{d_{b}}\left(g_{0}, \dot{r}\right)}$, then $\alpha_{*}\left(B g_{0}, B g_{1}\right) \geq 1$. As $\alpha_{*}\left(B g_{0}, B g_{1}\right) \geq 1, g_{1} \in B g_{0}$ and $g_{2} \in B g_{1}$, so $\alpha\left(g_{1}, g_{2}\right) \geq$ 1. Let $g_{2}, \cdots, g_{j} \in \overline{B_{d_{b}}\left(g_{0}, r\right)}$ for some $j \in \mathbb{N}$. As
$\alpha_{*}\left(B g_{1}, B g_{2}\right) \geq 1$, we have $\alpha\left(g_{2}, g_{3}\right) \geq 1$, which further implies $\alpha_{*}\left(B g_{2}, B g_{3}\right) \geq 1$. Continuing this process, we have $\alpha_{*}\left(B g_{j-1}, B g_{j}\right) \geq 1$. Now, by using Lemma 8

$$
\begin{aligned}
d_{b}\left(g_{j}, g_{j+1}\right) & \leq H_{d_{b}}\left(B g_{j-1}, B g_{j}\right) \leq \alpha_{*}\left(B g_{j-1}, B g_{j}\right) H_{d_{b}}\left(B g_{j-1}, B g_{j}\right) \leq \psi\left(D_{b}\left(g_{j-1}, g_{j}\right)\right) \\
& =\psi\left(\max \left\{d_{b}\left(g_{j-1}, g_{j}\right), \frac{d_{b}\left(g_{j-1}, B g_{j-1}\right) \cdot d_{b}\left(g_{j}, B g_{j}\right)}{\bar{a}+d_{b}\left(g_{j-1}, g_{j}\right)}, d_{b}\left(g_{j-1}, B g_{j-1}\right), d_{b}\left(g_{j}, B g_{j}\right)\right\}\right) \\
& =\psi\left(\max \left\{d_{b}\left(g_{j-1}, g_{j}\right), \frac{d_{b}\left(g_{j-1}, g_{j}\right) \cdot d_{b}\left(g_{j}, g_{j+1}\right)}{\bar{a}+d_{b}\left(g_{j-1}, g_{j}\right)}, d_{b}\left(g_{j-1}, g_{j}\right), d_{b}\left(g_{j}, g_{j+1}\right)\right\}\right) \\
& =\psi\left(\max \left\{d_{b}\left(g_{j-1}, g_{j}\right), d_{b}\left(g_{j}, g_{j+1}\right)\right\}\right) .
\end{aligned}
$$

If $\max \left\{d_{b}\left(g_{j-1}, g_{j}\right), d_{b}\left(g_{j}, g_{j+1}\right)\right\}=d_{b}\left(g_{j}, g_{j+1}\right)$, then $d_{b}\left(g_{j}\right.$, $\left.g_{j+1}\right) \leq \psi\left(d_{b}\left(g_{j}, g_{j+1}\right)\right)$. This is contradiction to the fact that $\psi(u)<u$ for all $u>0$. Hence, we obtain $\max \left\{d_{b}\left(g_{j-1}\right.\right.$, $\left.\left.g_{j}\right), d_{b}\left(g_{j}, g_{j+1}\right)\right\}=d_{b}\left(g_{j-1}, g_{j}\right)$. Therefore, we have

$$
\begin{aligned}
d_{b}\left(g_{j}, g_{j+1}\right) & \leq \psi\left(d_{b}\left(g_{j-1}, g_{j}\right)\right) \leq \cdots \\
& \leq \psi^{j}\left(d_{b}\left(g_{0}, g_{1}\right)\right)
\end{aligned}
$$

Now, by using triangular inequality and by (10), we have

$$
\begin{aligned}
d_{b}\left(g_{0}, g_{j+1}\right) \leq & t d_{b}\left(g_{0}, g_{1}\right)+t^{2} d_{b}\left(g_{1}, g_{2}\right)+\cdots \\
& +t^{j+1} d_{b}\left(g_{j}, g_{j+1}\right) \\
\leq & t d_{b}\left(g_{0}, g_{1}\right)+t^{2} \psi\left(d_{b}\left(g_{0}, g_{1}\right)\right)+\cdots \\
& +t^{j+1} \psi^{j}\left(d_{b}\left(g_{0}, g_{1}\right)\right) \\
\leq & \sum_{i=0}^{j} t^{i+1}\left\{\psi^{i}\left(d_{b}\left(g_{0}, g_{1}\right)\right)\right\} \leq \dot{r} .
\end{aligned}
$$

Thus $g_{j+1} \in \overline{B_{d_{b}}\left(g_{0}, \dot{r}\right)}$. Hence, by induction, $g_{n} \in \overline{B_{d_{b}}\left(g_{0}, \dot{r}\right)}$. As $\alpha_{*}\left(B g_{j-1}, B g_{j}\right) \geq 1, g_{j} \in B g_{j}, g_{j+1} \in B g_{j}$, then we have $\alpha\left(g_{j}, g_{j+1}\right) \geq 1$. Also $B$ is semi- $\alpha_{*}-$ admissible multifunction on $\overline{B_{d_{b}}\left(g_{0}, \dot{r}\right)}$, and therefore $\alpha_{*}\left(B g_{j}, B g_{j+1}\right) \geq 1$. This further implies that $\alpha\left(g_{j+1}, g_{j+2}\right) \geq 1$. Continuing this process, we have $\alpha\left(g_{n}, g_{n+1}\right) \geq 1$ for all $n \in \mathbb{N}$. Now, inequality (10) can be written as

$$
d_{b}\left(g_{n}, g_{n+1}\right) \leq \psi^{n}\left(d_{b}\left(g_{0}, g_{1}\right)\right) \quad \forall n \in \mathbb{N} .
$$

Fix $\in>0$ and let $k_{1}(\epsilon) \in \mathbb{N}$, such that

$$
\sum_{k \geq k_{1}(\epsilon)} t^{k} \psi^{k}\left(d_{b}\left(g_{0}, g_{1}\right)\right)<\epsilon
$$

Let $n, m \in \mathbb{N}$ with $m>n>k_{1}(\epsilon)$. Now, we have

$$
\begin{aligned}
d_{b}\left(g_{n}, g_{m}\right) & \leq \sum_{k=n}^{m-1} d_{b}\left(g_{k}, g_{k+1}\right) \\
& \leq \sum_{k=n}^{m-1} t^{k} \psi^{k}\left(d_{b}\left(g_{0}, g_{1}\right)\right), \text { by }(12) \\
d_{b}\left(g_{n}, g_{m}\right) & \leq \sum_{k \geq k_{1}(\epsilon)} t^{k} \psi^{k}\left(d_{b}\left(g_{0}, g_{1}\right)\right)<\epsilon .
\end{aligned}
$$

Thus, $\left\{M B\left(g_{n}\right)\right\}$ is a Cauchy sequence in $\left(\overline{B_{d_{b}}\left(g_{0}, r\right)}, d_{b}\right)$. As every closed ball in a complete D.B.M space is complete, there exist $g^{*} \in \overline{B_{d_{b}}\left(g_{0}, \dot{r}\right)}$ such that $\left\{M B\left(g_{n}\right)\right\} \longrightarrow g^{*}$, and

$$
\lim _{n \longrightarrow \infty} d_{b}\left(g_{n}, g^{*}\right)=0
$$

By assumption, we have $\alpha\left(g_{n}, g^{*}\right) \geq 1$ for all $n \in \mathbb{N} \cup\{0\}$. Thus, $\alpha_{*}\left(B g_{n}, B g^{*}\right) \geq 1$. Now, we have

$$
\begin{aligned}
d_{b}\left(g^{*}, B g^{*}\right) \leq t d_{b}\left(g^{*}, g_{n+1}\right)+t d_{b}\left(g_{n+1}, B g^{*}\right) \\
\leq t d_{b}\left(g^{*}, g_{n+1}\right)+t H_{d_{b}}\left(B g_{n}, B g^{*}\right) \quad \text { by Lemma } 8 \\
\leq t d_{b}\left(g^{*}, g_{n+1}\right)+t\left\{\alpha_{*}\left(B g_{n}, B g^{*}\right) H_{d_{b}}\left(B g_{n}, B g^{*}\right)\right\} \\
\leq t d_{b}\left(g^{*}, g_{n+1}\right)+t \psi\left(\operatorname { m a x } \left\{d_{b}\left(g_{n}, g^{*}\right),\right.\right. \\
\quad \frac{d_{b}\left(g_{n}, B g_{n}\right) \cdot d_{b}\left(g^{*}, B g^{*}\right)}{\bar{a}+d_{b}\left(g_{n}, g^{*}\right)}, d_{b}\left(g_{n}, B g_{n}\right), \\
\left.\left.\quad d_{b}\left(g^{*}, B g^{*}\right)\right\}\right) \\
\leq t d_{b}\left(g^{*}, g_{n+1}\right)+t \psi\left(\operatorname { m a x } \left\{d_{b}\left(g_{n}, g^{*}\right),\right.\right.
\end{aligned}
$$




$$
\begin{aligned}
& \frac{d_{b}\left(g_{n}, g_{n+1}\right) \cdot d_{b}\left(g^{*}, B g^{*}\right)}{\bar{a}+d_{b}\left(g_{n}, g^{*}\right)}, d_{b}\left(g_{n}, g_{n+1}\right), \\
& \left.\left.d_{b}\left(g^{*}, B g^{*}\right)\right\}\right) .
\end{aligned}
$$

Letting $n \longrightarrow \infty$ and by using inequality (15), we obtain (1 t) $d_{b}\left(g^{*}, B g^{*}\right) \leq 0$. So $(1-t) \neq 0$, and then $d_{b}\left(g^{*}, B g^{*}\right)=0$. Hence $g^{*} \in B g^{*}$. So $B$ has a fixed point in $\overline{B_{d_{b}}\left(g_{0}, r\right)}$.

Let $M$ be a nonempty set. Then $\left(M, \preceq, d_{b}\right)$ is called a preordered D.B.M space if $d_{b}$ is called D.B.M on $M$. Let $\left(M, \preceq, d_{b}\right)$ be a preordered D.B.M space and $H, K \subseteq M$. We say that $H \preceq_{\dot{r}} K$ whenever for each $\bar{a} \in H$ there exist $b \in K$ such that $\bar{a} \preceq b$. Also, we say that $H \preceq_{\dot{r}} K$ whenever, for each $\bar{a} \in H$ and $b \in K$, we have $\bar{a} \preceq b$.

Corollary 10. Let $\left(M, \preceq, d_{b}\right)$ be a preordered complete D.B.M space, $\dot{r}>0, g_{0} \in \overline{B_{d_{b}}\left(g_{0}, \dot{r}\right)}$, and $B: M \longrightarrow P(M)$ be a multifunction on $\overline{B_{d_{b}}\left(g_{0}, \dot{r}\right)} ;\left\{M B\left(g_{n}\right)\right\}$ is a sequence generated by $g_{0}$, with $g_{0} \preceq g_{1}$. Assume that, for some $\psi \in \Psi$ and

$$
\begin{aligned}
& D_{b}(g, q)=\max \left\{d_{b}(g, q), \frac{d_{b}(g, B g) \cdot d_{b}(q, B q)}{\bar{a}+d_{b}(g, q)},\right. \\
& \left.d_{b}(g, B g), d_{b}(q, B q)\right\}
\end{aligned}
$$

where $\bar{a}>0$, the following hold:

$$
\begin{gathered}
H_{d_{b}}(B g, B q) \leq \psi\left(D_{b}(g, q)\right) \\
\forall g, q \in \overline{B_{d_{b}}\left(g_{0}, \dot{r}\right)} \cap\left\{M B\left(g_{n}\right)\right\} \text { with } g \preceq q \\
\text { and } \sum_{i=0}^{n} t^{i+1}\left\{\psi^{i}\left(d_{b}\left(g_{0}, g_{1}\right)\right)\right\} \leq \dot{r} \quad \forall n \in \mathbb{N} \cup\{0\} .
\end{gathered}
$$

If $g, q \in \overline{B_{d_{b}}\left(g_{0}, \dot{r}\right)}$, such that $g \preceq q$ implies $B g \preceq_{\dot{r}} B q$. Then $\left\{M B\left(g_{n}\right)\right\}$ is a sequence in $\overline{B_{d_{b}}\left(g_{0}, r\right)}, g_{n} \preceq g_{n+1}$, and $\left\{M B\left(g_{n}\right)\right\} \longrightarrow g^{*} \in \overline{B_{d_{b}}\left(g_{0}, \dot{r}\right)}$. Also if $g^{*} \preceq g_{n}$ or $g_{n} \preceq g^{*}$, for all $n \in \mathbb{N} \cup\{0\}$, and inequality (18) holds for all $g, q \in$ $\overline{B_{d_{b}}\left(g_{0}, \dot{r}\right)} \cap\left\{M B\left(g_{n}\right)\right\} \cup\left\{g^{*}\right\}$. Then $g^{*}$ is a fixed point of $B$ in $\overline{B_{d_{b}}\left(g_{0}, \dot{r}\right)}$.

Corollary 11. Let $\left(M, \preceq, d_{b}\right)$ be a preordered complete D.B.M space, $\dot{r}>0, g_{0} \in \overline{B_{d_{b}}\left(g_{0}, \dot{r}\right)}$, and $B: M \longrightarrow P(M)$ be a multifunction on $\overline{B_{d_{b}}\left(g_{0}, \dot{r}\right)} ;\left\{M B\left(g_{n}\right)\right\}$ is a sequence generated by $g_{0}$, with $g_{0} \preceq g_{1}$. Assume that, for some $k \in[0,1)$ and

$$
\begin{aligned}
& D_{b}(g, q)=\max \left\{d_{b}(g, q), \frac{d_{b}(g, B g) \cdot d_{b}(q, B q)}{\bar{a}+d_{b}(g, q)},\right. \\
& \left.d_{b}(g, B g), d_{b}(q, B q)\right\}
\end{aligned}
$$

where $\bar{a}>0$, the following hold:

$$
H_{d_{b}}(B g, B q) \leq k\left(D_{b}(g, q)\right)
$$$$
\forall g, q \in \overline{B_{d_{b}}\left(g_{0}, \dot{r}\right)} \cap\left\{M B\left(g_{n}\right)\right\} \text { with } g \preceq q
$$

$$
\text { and } \sum_{i=0}^{n} t^{i+1}\left\{k^{i}\left(d_{b}\left(g_{0}, g_{1}\right)\right)\right\} \leq \dot{r} \quad \forall n \in \mathbb{N} \cup\{0\} \text {. }
$$

If $g, q \in \overline{B_{d_{b}}\left(g_{0}, \dot{r}\right)}$, such that $g \preceq q$ implies $B g \unlhd_{r} B q$. Then $\left\{M B\left(g_{n}\right)\right\}$ is a sequence in $\overline{B_{d_{b}}\left(g_{0}, r\right)}, g_{n} \preceq g_{n+1}$, and $\left\{M B\left(g_{n}\right)\right\} \longrightarrow g^{*} \in \overline{B_{d_{b}}\left(g_{0}, \dot{r}\right)}$. Also if $g^{*} \preceq g_{n}$ or $g_{n} \preceq g^{*}$, for all $n \in \mathbb{N} \cup\{0\}$, and inequality (21) holds for all $g, q \in$ $\overline{B_{d_{b}}\left(g_{0}, \dot{r}\right)} \cap\left\{M B\left(g_{n}\right)\right\} \cup\left\{g^{*}\right\}$. Then $g^{*}$ is a fixed point of $B$ in $\overline{B_{d_{b}}\left(g_{0}, r\right)}$.

Corollary 12. Let $\left(M, \preceq, d_{l}\right)$ be a preordered D.M space, $\dot{r}>$ $0, g_{0} \in \overline{B_{d_{l}}\left(g_{0}, \dot{r}\right)}$, and $B: M \longrightarrow P(M)$ be a multifunction on $\overline{B_{d_{b}}\left(g_{0}, \dot{r}\right)} ;\left\{M B\left(g_{n}\right)\right\}$ is a sequence generated by $g_{0}$, with $g_{0} \preceq g_{1}$. Assume that, for some $\psi \in \Psi$ and

$$
\begin{aligned}
& D_{l}(g, q)=\max \left\{d_{l}(g, q), \frac{d_{l}(g, B g) \cdot d_{l}(q, B q)}{\bar{a}+d_{l}(g, q)}\right. \\
& \left.d_{l}(g, B g), d_{l}(q, B q)\right\}
\end{aligned}
$$

where $\bar{a}>0$, the following hold:

$$
\begin{gathered}
H_{d_{l}}(B g, B q) \leq \psi\left(D_{l}(g, q)\right) \\
\forall g, q \in \overline{B_{d_{l}}\left(g_{0}, \dot{r}\right)} \cap\left\{M B\left(g_{n}\right)\right\} \text { with } g \preceq q \\
\text { and } \sum_{i=0}^{n} \psi^{i}\left(d_{l}\left(g_{0}, g_{1}\right)\right) \leq \dot{r} \quad \forall n \in \mathbb{N} \cup\{0\} .
\end{gathered}
$$

If $g, q \in \overline{B_{d_{l}}\left(g_{0}, \dot{r}\right)}$, such that $g \preceq q$ implies $B g \preceq_{r} B q$. Then $\left\{M B\left(g_{n}\right)\right\}$ is a sequence in $\overline{B_{d_{l}}\left(g_{0}, \dot{r}\right)}, g_{n} \preceq g_{n+1}$, and $\left\{M B\left(g_{n}\right)\right\} \longrightarrow g^{*} \in \overline{B_{d_{l}}\left(g_{0}, \dot{r}\right)}$. Also if $g^{*} \preceq g_{n}$ or $g_{n} \preceq g^{*}$, for all $n \in \mathbb{N} \cup\{0\}$, and inequality (24) holds for all $g, q \in$ $\overline{B_{d_{l}}\left(g_{0}, r\right)} \cap\left\{M B\left(g_{n}\right)\right\} \cup\left\{g^{*}\right\}$. Then $g^{*}$ is a fixed point of $B$ in $\overline{B_{d_{l}}\left(g_{0}, \dot{r}\right)}$.

Corollary 13. Let $\left(M, \preceq, d_{l}\right)$ be a preordered complete D.M space, $\dot{r}>0, g_{0} \in \overline{B_{d_{l}}\left(g_{0}, \dot{r}\right)}$, and $B: M \longrightarrow P(M)$ be a multifunction on $\overline{B_{d_{b}}\left(g_{0}, \dot{r}\right)} ;\left\{M B\left(g_{n}\right)\right\}$ is a sequence generated by $g_{0}$, with $g_{0} \preceq g_{1}$. Assume that, for some $k \in[0,1)$ and

$$
\begin{aligned}
& D_{l}(g, q)=\max \left\{d_{l}(g, q), \frac{d_{l}(g, B g) \cdot d_{l}(q, B q)}{\bar{a}+d_{l}(g, q)},\right. \\
& \left.d_{l}(g, B g), d_{l}(q, B q)\right\}
\end{aligned}
$$


where $\bar{a}>0$, the following hold:

$$
\begin{aligned}
& H_{d_{l}}(B g, B q) \leq k\left(D_{l}(g, q)\right) \\
& \forall g, q \in \overline{B_{d_{l}}\left(g_{0}, \dot{r}\right)} \cap\left\{M B\left(g_{n}\right)\right\} \text { with } g \preceq q
\end{aligned}
$$

$$
\text { and } \sum_{i=0}^{n} k^{i}\left(d_{l}\left(g_{0}, g_{1}\right)\right) \leq \dot{r} \quad \forall n \in \mathbb{N} \cup\{0\} \text {. }
$$

If $g, q \in \overline{B_{d_{l}}\left(g_{0}, \dot{r}\right)}$, such that $g \preceq q$ implies $B g \preceq_{r} B q$. Then $\left\{M B\left(g_{n}\right)\right\}$ is a sequence in $\overline{B_{d_{l}}\left(g_{0}, r\right)}, g_{n} \preceq g_{n+1}$, and $\left\{M B\left(g_{n}\right)\right\} \longrightarrow g^{*} \in \overline{B_{d_{l}}\left(g_{0}, r\right)}$. Also if $g^{*} \preceq g_{n}$ or $g_{n} \preceq g^{*}$, for all $n \in \mathbb{N} \cup\{0\}$, and inequality (27) holds for all $g, q \in$ $\overline{B_{d_{l}}\left(g_{0}, r\right)} \cap\left\{M B\left(g_{n}\right)\right\} \cup\left\{g^{*}\right\}$. Then $g^{*}$ is a fixed point of $B$ in $\overline{B_{d_{l}}\left(g_{0}, r\right)}$.

Example 14. Let $M=Q^{+} \cup\{0\}$ and let $d_{b}: M \times M \longrightarrow M$ be the D.B.M space on $M$ defined by

$$
d_{b}(g, q)=(g+q)^{2} \quad \forall g, q \in M
$$

with parameter $t>1$. Define the multivalued mappings, $B$ : $M \times M \longrightarrow P(M)$ by

$$
B g= \begin{cases}{\left[\frac{g}{3}, \frac{2}{3} g\right]} & \text { if } g \in[0,9] \cap M \\ {[g, g+1]} & \text { if } g \in(9, \infty) \cap M\end{cases}
$$

Considering $g_{0}=1, \dot{r}=100$, and $\bar{a}=1, b=2$, then $\overline{B_{d_{b}}\left(g_{0}, r\right)}=[0,9] \cap M$. Now $d_{b}\left(g_{0}, B g_{0}\right)=d_{b}(1$, $B 1)=d_{b}(1,1 / 3)=16 / 9$. So we obtain a sequence $\left\{M B\left(g_{n}\right)\right\}=\{1,1 / 3,1 / 9,1 / 27, \ldots\}$ in $M$ generated by $g_{0}$. Let $t=1.2, \psi(t)=4 t / 5$, and then $t \psi(t)<t$. Define

$$
\alpha(g, q)= \begin{cases}1 & \text { if } g, q \in[0,9] \cap M \\ \frac{3}{2} & \text { otherwise. }\end{cases}
$$

Now,

$$
\begin{gathered}
\alpha_{*}(B 10, B 11) H_{d_{b}}(B 10, B 11)=\left(\frac{3}{2}\right)(484) \\
>\psi\left(D_{b}(g, q)\right)=\frac{4}{5}(484) .
\end{gathered}
$$

So the contractive condition does not hold on $M$. Now, for all $g, q \in \overline{B_{d_{b}}\left(g_{0}, \dot{r}\right)} \cap\left\{M B\left(g_{n}\right)\right\}$, we have

$$
\begin{gathered}
\alpha_{*}(B g, B q) H_{d_{b}}(B g, B q)=1\left[\operatorname { m a x } \left\{\sup _{\bar{a} \in B g} d_{b}(\bar{a}, B q),\right.\right. \\
\left.\left.\sup _{b \in B q} d_{b}(B g, b)\right\}\right]=\max \left\{\sup _{\bar{a} \in B g} d_{b}\left(\bar{a},\left[\frac{q}{3}, \frac{2 q}{3}\right]\right),\right. \\
\left.\sup _{b \in B q} d_{b}\left(\left[\frac{g}{3}, \frac{2 g}{3}\right], b\right)\right\}=\max \left\{\operatorname { s u p } _ { \overline { a } \in B g } d _ { b } \left(\frac{2 g}{3},\right.\right. \\
\left.\left.\left[\frac{q}{3}, \frac{2 q}{3}\right]\right), \sup _{b \in B q} d_{b}\left(\left[\frac{g}{3}, \frac{2 g}{3}\right], \frac{2 q}{3}\right)\right\}
\end{gathered}
$$

$$
\begin{aligned}
& =\max \left\{d_{b}\left(\frac{2 g}{3}, \frac{q}{3}\right), d_{b}\left(\frac{g}{3}, \frac{2 q}{3}\right)\right\} \\
& =\max \left\{\frac{(2 g+q)^{2}}{9}, \frac{(g+2 q)^{2}}{9}\right\} \\
& \leq \psi\left(\operatorname { m a x } \left\{(g+q)^{2}, \frac{256 g^{2} q^{2}}{81\left\{1+(g+q)^{2}\right\}}, \frac{16 g^{2}}{9},\right.\right. \\
& \left.\left.\frac{16 q^{2}}{9}\right\}\right)=\psi\left(d_{b}(g, q)\right) .
\end{aligned}
$$

So the contractive condition holds on $\overline{B_{d_{b}}\left(\dot{c}_{0}, \dot{r}\right)} \cap\left\{M B\left(g_{n}\right)\right\}$. As $t=1.2>1$, then

$$
\begin{aligned}
& \sum_{i=0}^{n} t^{i+1}\left\{\psi^{i}\left(d_{b}\left(g_{0}, g_{1}\right)\right)\right\}=\frac{16}{9} \times \frac{6}{5} \sum_{i=0}^{n}\left(\frac{24}{25}\right)^{i}<100 \\
& =\dot{r} .
\end{aligned}
$$

Hence, all the conditions of Theorem 9 are satisfied. Now, we have that $\left\{M B\left(g_{n}\right)\right\}$ is a sequence in $\overline{B_{d_{b}}\left(g_{0}, r\right)}, \alpha\left(g_{n}, g_{n+1}\right) \geq$ 1 and $\left\{M B\left(g_{n}\right)\right\} \longrightarrow 0 \in \overline{B_{d_{b}}\left(g_{0}, r\right)}$. Moreover, 0 is a fixed point of $B$.

\section{Fixed Point Results For Graphic Contractions}

In this section, we present an application of Theorem 9 in graph theory. Jachymski [27] proved the result concerning contraction mappings on metric space with a graph. Hussain et al. [28] discussed the fixed points theorem for graphic contraction and gave an application. A graph $\dot{G}$ is affix if there is a way among any two vertices (see for details $[29,30]$ ).

Definition 15. Let $M$ be a nonempty set and $\dot{G}=(V(\dot{G}), Q(G))$ be a graph such that $V(\dot{G})=M$, and $B: M \longrightarrow P(M)$ is said to be multigraph preserving if $(g, q) \in Q(G)$, and then $(w, p) \in Q(G)$ for all $w \in B g$ and $p \in B q$.

Theorem 16. Let $\left(M, d_{b}\right)$ be a complete D.B.M space endowed with a graph $\dot{G}$. Suppose there exists a function $\alpha: M \times M \longrightarrow$ $[0, \infty)$. Let, $\dot{r}>0, g_{0} \in \overline{B_{d_{b}}\left(g_{0}, \dot{r}\right)}, B: M \longrightarrow P(M)$, and let for a sequence $\left\{M B\left(g_{n}\right)\right\}$ in $M$ generated by $g_{0}$, with $\left(g_{0}, g_{1}\right) \in$ $Q(G)$. Suppose that the following are satisfy:

(i) $B$ is a graph preserving for all $g, q \in \overline{B_{d_{b}}\left(g_{0}, r\right)} \cap$ $\left\{M B\left(g_{n}\right)\right\}$;

(ii) there exists $\psi \in \Psi$ and

$$
\begin{aligned}
& D_{b}(g, q)=\max \left\{d_{b}(g, q), \frac{d_{l}(g, B g) \cdot d_{l}(q, B q)}{\bar{a}+d_{l}(g, q)}\right. \\
& \left.d_{b}(g, B g), d_{b}(q, B q)\right\}
\end{aligned}
$$


where $\bar{a}>0$ such that

$$
H_{d_{b}}(B g, B q) \leq \psi\left(D_{b}(g, q)\right)
$$

for all $g, q \in \overline{B_{d_{b}}\left(g_{0}, \dot{r}\right)} \cap\left\{M B\left(g_{n}\right)\right\}$ and $(g, q) \in Q(G)$; $t>1$.

(iii) $\sum_{i=0}^{n} t^{i+1}\left\{\psi^{i}\left(d_{b}\left(g_{0}, B g_{0}\right)\right)\right\} \leq \dot{r}$ for all $n \in \mathbb{N} \cup\{0\}$ and

Then, $\left\{M B\left(g_{n}\right)\right\}$ is a sequence in $\overline{B_{d_{b}}\left(g_{0}, r\right)},\left(g_{n}, g_{n+1}\right) \in$ $Q(G)$ and $\left\{M B\left(g_{n}\right)\right\} \longrightarrow g^{*}$. Also, if and inequality (36) holds for $g^{*}$ and $\left(g_{n}, g^{*}\right) \in Q(G)$ or $\left(g^{*}, g_{n}\right) \in Q(G)$ for all $n \in$ $\mathbb{N} \cup\{0\}$, then $B$ has a fixed point $g^{*}$ in $\overline{B_{d_{b}}\left(g_{0}, \dot{r}\right)}$.

Proof. Define, $\alpha: M \times M \longrightarrow[0, \infty)$ by

$$
\alpha(g, q)= \begin{cases}1, & \text { if } g \in \overline{B_{d_{b}}\left(g_{0}, r\right)},(g, q) \in Q(G) \\ 0, & \text { otherwise. }\end{cases}
$$

As $\left\{M B\left(g_{n}\right)\right\}$ is a sequence in $M$ generated by $g_{0}$ with $\left(g_{0}, g_{1}\right) \in Q(G)$, we have $\alpha\left(g_{0}, g_{1}\right) \geq 1$. Let $\alpha(g, q) \geq 1$, and then $(g, q) \in Q(G)$. From (i), we have $(w, p) \in Q(G)$ for all $w \in B g$ and $p \in B q$. This implies that $\alpha(w, p)=1$ for all $w \in$ $B g$ and $p \in B q$. This implies that $\inf \{\alpha(w, p): w \in B g, p \in$ $B q\}=1$. So, $B: M \longrightarrow P(M)$ is a semi- $\alpha_{*}$-admissible multifunction on $\overline{B_{d_{b}}\left(g_{0}, \dot{r}\right)}$. Moreover, inequality (36) can be written as

$$
\alpha_{*}(B g, B q) H_{d_{b}}(B g, B q) \leq \psi\left(D_{b}(g, q)\right)
$$

for all elements $g, q$ in $\overline{B_{d_{b}}\left(g_{0}, r\right)} \cap\left\{M B\left(g_{n}\right)\right\}$ with either $\alpha(g, q) \geq 1$ or $\alpha(q, g) \geq 1$. Also, (iii) holds. Then, by Theorem 9, we have that $\left\{M B\left(g_{n}\right)\right\}$ is a sequence in $\overline{B_{d_{b}}\left(g_{0}, r\right)}$ and $\left\{M B\left(g_{n}\right)\right\} \longrightarrow g^{*} \in \overline{B_{d_{b}}\left(g_{0}, r\right)}$. Now, $g_{n}, g^{*} \in \overline{B_{d_{b}}\left(g_{0}, \dot{r}\right)}$ and either $\left(g_{n}, g^{*}\right) \in Q(G)$ or $\left(g^{*}, g_{n}\right) \in Q(G)$ for all $n \in \mathbb{N} \cup\{0\}$, and inequality (36) holds for all $g, q \in$ $\overline{B_{d_{b}}\left(g_{0}, \dot{r}\right)} \cap\left\{M B\left(g_{n}\right)\right\} \cup\left\{g^{*}\right\}$. Then we have $\alpha\left(g_{n}, g^{*}\right) \geq 1$ or $\alpha\left(g^{*}, g_{n}\right) \geq 1$ for all $n \in \mathbb{N} \cup\{0\}$ and inequality (5) holds for all $g, q \in \overline{B_{d_{b}}\left(g_{0}, \dot{r}\right)} \cap\left\{M B\left(g_{n}\right)\right\} \cup\left\{g^{*}\right\}$. So, all the conditions of Theorem 9 are satisfied. Hence, by Theorem $9, B$ has a common fixed point $g^{*}$ in $\overline{B_{d_{b}}\left(g_{0}, \dot{r}\right)}$ and $d_{b}\left(g^{*}, g^{*}\right)=0$.

\section{Fixed Point Results for Single-Valued Mapping}

In this section we discussed some fixed point results for selfmapping in complete D.B.M space. Let $\left(M, d_{b}\right)$ be a D.B.M space, $g_{0} \in M$, and $B: M \longrightarrow M$ be a mapping. Let $g_{1}=B g_{0}$, $g_{2}=B g_{1}$. Continuing this process, we construct a sequence $g_{n}$ of points in $M$ such that $g_{n+1}=B_{g_{n}}$. We denote this iterative sequence by $\left\{g_{n}\right\}$. We say that $\left\{g_{n}\right\}$ is a sequence in $M$ generated by $g_{0}$.

Theorem 17. Let $\left(M, d_{b}\right)$ be a complete D.B.M space, $\dot{r}>$ $0, g_{0} \in \overline{B_{d_{b}}\left(g_{0}, \dot{r}\right)}$, and $B: M \longrightarrow M$ be a semi- $\alpha$-admissible function on $\overline{B_{d_{b}}\left(g_{0}, r\right)} ;\left\{g_{n}\right\}$ is a sequence in $M$ generated by $g_{0}, \alpha\left(g_{0}, g_{1}\right) \geq 1$. Assume that, for some $\psi \in \Psi$ and

$$
\begin{aligned}
& D_{b}(g, q)=\max \left\{d_{b}(g, q), \frac{d_{b}(g, B g) \cdot d_{b}(q, B q)}{\bar{a}+d_{b}(g, q)},\right. \\
& \left.d_{b}(g, B g), d_{b}(q, B q)\right\}
\end{aligned}
$$

where $\bar{a}>0$, the following hold:

$$
\begin{aligned}
& \alpha(B g, B q) H_{d_{b}}(B g, B q) \leq\left(D_{b}(g, q)\right) \\
& \forall g, q \in \overline{B_{d_{b}}\left(g_{0}, \dot{r}\right)} \cap\left\{g_{n}\right\} \\
& \sum_{i=0}^{n} B^{i+1}\left\{\psi^{i}\left(d_{b}\left(g_{0}, g_{1}\right)\right)\right\} \leq \dot{r} \quad \forall n \in \mathbb{N} \cup\{0\} .
\end{aligned}
$$

Then, $\left\{g_{n}\right\}$ is a sequence in $\overline{B_{d_{b}}\left(g_{0}, r\right)}, \alpha\left(g_{n}, g_{n+1}\right) \geq 1$, and $\left\{g_{n}\right\} \longrightarrow g^{*} \in \overline{B_{d_{b}}\left(g_{0}, \dot{r}\right)}$. Also if $\alpha\left(g_{n}, g^{*}\right) \geq 1$ or $\alpha\left(g^{*}, g_{n}\right) \geq$ 1 , for all $n \in \mathbb{N} \cup\{0\}$, and inequality (40) holds for all $g, q \in$ $\overline{B_{d_{b}}\left(g_{0}, \dot{r}\right)} \cap\left\{g_{n}\right\} \cup\left\{g^{*}\right\}$. Then $B$ has a common fixed point $g^{*}$ in $\overline{B_{d_{b}}\left(g_{0}, r\right)}$.

Proof. The proof of the above theorem is similar to Theorem 17.

Corollary 18. Let $\left(M, \preceq, d_{b}\right)$ be a preordered complete D.B.M space, $\dot{r}>0, g_{0} \in \overline{B_{d_{b}}\left(g_{0}, r\right)}$, and $B: M \longrightarrow M$ be a selfmapping on $\overline{B_{d_{b}}\left(g_{0}, \dot{r}\right)} ;\left\{g_{n}\right\}$ is a sequence generated by $g_{0}$, with $g_{0} \preceq g_{1}$. Assume that, for some $k \in[0,1)$ and

$$
\begin{aligned}
& D_{b}(g, q)=\max \left\{d_{b}(g, q), \frac{d_{b}(g, B g) \cdot d_{b}(q, B q)}{\bar{a}+d_{b}(g, q)},\right. \\
& \left.d_{b}(g, B g), d_{b}(q, B q)\right\}
\end{aligned}
$$

where $\bar{a}>0$, the following hold:

$$
\begin{aligned}
& H_{d_{b}}(B g, B q) \leq k\left(D_{b}(g, q)\right) \\
& \forall g, q \in \overline{B_{d_{b}}\left(g_{0}, \dot{r}\right)} \cap\left\{g_{n}\right\} \text { with } g \preceq q
\end{aligned}
$$

$$
\text { and } \sum_{i=0}^{n} t^{i+1}\left\{k^{i}\left(d_{b}\left(g_{0}, g_{1}\right)\right)\right\} \leq \dot{r} \quad \forall n \in \mathbb{N} \cup\{0\} \text {. }
$$

If $g, q \in \overline{B_{d_{b}}\left(g_{0}, \dot{r}\right)}$, such that $g \preceq q$ implies $B g \preceq_{\dot{r}} B q$. Then $\left\{g_{n}\right\}$ is a sequence in $\overline{B_{d_{b}}\left(g_{0}, \dot{r}\right)}, g_{n} \preceq g_{n+1}$, and $\left\{g_{n}\right\} \longrightarrow g^{*} \epsilon$ $\overline{B_{d_{b}}\left(g_{0}, \dot{r}\right)}$. Also if $g^{*} \preceq g_{n}$ or $g_{n} \preceq g^{*}$, for all $n \in \mathbb{N} \cup\{0\}$, and inequality (43) holds for all $g, q \in \overline{B_{d_{b}}\left(g_{0}, \dot{r}\right)} \cap\left\{g_{n}\right\} \cup\left\{g^{*}\right\}$. Then $g^{*}$ is a fixed point of $B$ in $\overline{B_{d_{b}}\left(g_{0}, \dot{r}\right)}$.

Corollary 19. Let $\left(M, \preceq, d_{l}\right)$ be a preordered complete D.M space, $\dot{r}>0, g_{0} \in \overline{B_{d_{l}}\left(g_{0}, \dot{r}\right)}$, and $B: M \longrightarrow M$ be $a$ 
self-mapping on $\overline{B_{d_{l}}\left(g_{0}, \dot{r}\right)} ;\left\{g_{n}\right\}$ is a sequence generated by $g_{0}$, with $g_{0} \preceq g_{1}$. Assume that, for some $\psi \in \Psi$ and

$$
\begin{aligned}
& D_{l}(g, q)=\max \left\{d_{l}(g, q), \frac{d_{l}(g, B g) \cdot d_{l}(q, B q)}{\bar{a}+d_{l}(g, q)},\right. \\
& \left.d_{l}(g, B g), d_{l}(q, B q)\right\}
\end{aligned}
$$

where $\bar{a}>0$, the following hold:

$$
\begin{aligned}
& H_{d_{l}}(B g, B q) \leq \psi\left(D_{l}(g, q)\right) \\
& \forall g, q \in \overline{B_{d_{l}}\left(g_{0}, \dot{r}\right)} \cap\left\{g_{n}\right\} \text { with } g \preceq q
\end{aligned}
$$

and $\sum_{i=0}^{n} \psi^{i}\left(d_{l}\left(g_{0}, g_{1}\right)\right) \leq \dot{r} \quad \forall n \in \mathbb{N} \cup\{0\}$.

If $g, q \in \overline{B_{d_{l}}\left(g_{0}, \dot{r}\right)}$, such that $g \preceq q$ implies $B g \unlhd_{\dot{r}} B q$. Then $\left\{g_{n}\right\}$ is a sequence in $\overline{B_{d_{l}}\left(g_{0}, \dot{r}\right)}, g_{n} \preceq g_{n+1}$, and $\left\{g_{n}\right\} \longrightarrow g^{*} \in$ $\overline{B_{d_{l}}\left(g_{0}, \dot{r}\right)}$. Also if $g^{*} \preceq g_{n}$ or $g_{n} \preceq g^{*}$, for all $n \in \mathbb{N} \cup\{0\}$, and inequality (46) holds for all $g, q \in \overline{B_{d_{l}}\left(g_{0}, \dot{r}\right)} \cap\left\{g_{n}\right\} \cup\left\{g^{*}\right\}$. Then $g^{*}$ is a fixed point of $B$ in $\overline{B_{d_{l}}\left(g_{0}, \dot{r}\right) \text {. }}$

Recall that if $(M, \preceq)$ is a preordered set and $A: M \longrightarrow M$ is such that for $g, q \in M$, with $g \preceq q$ implying $A g \preceq A q$, then the mapping $A$ is said to be nondecreasing.

Corollary 20. Let $\left(M, \preceq, d_{l}\right)$ be a preordered complete D.M space, $\dot{r}>0, g_{0}$ be an arbitrary point in $\overline{B_{d_{l}}\left(g_{0}, \dot{r}\right)}, B$ : $M \longrightarrow M$ be a self-mapping on $\overline{B_{d_{l}}\left(g_{0}, \dot{r}\right)}$, and $\left\{g_{n}\right\}$ be a Picard sequence in $M$ with initial guess $g_{0}$, with $g_{0} \preceq g_{1}$. For some $k \in[0,1)$ and

$$
\begin{aligned}
& D_{l}(g, q)=\max \left\{d_{l}(g, q), \frac{d_{l}(g, B g) \cdot d_{l}(q, B q)}{\bar{a}+d_{l}(g, q)},\right. \\
& \left.d_{l}(g, B g), d_{l}(q, B q)\right\}
\end{aligned}
$$

where $\bar{a}>0$, the following hold:

$$
\begin{gathered}
d_{l}(B g, B q) \leq k\left(D_{l}(g, q)\right) \\
\forall g, q \in \overline{B_{d_{l}}\left(g_{0}, \dot{r}\right)} \cap\left\{g_{n}\right\} \text { with } g \preceq q \\
\text { and } \sum_{i=0}^{j} k^{i}\left(d_{l}\left(g_{0}, g_{1}\right)\right) \leq \dot{r} \quad \forall j \in \mathbb{N} \cup\{0\} .
\end{gathered}
$$

Then, $\left\{g_{n}\right\}$ is a sequence in $\overline{B_{d_{l}}\left(g_{0}, \dot{r}\right)}$, such that $g_{n} \preceq g_{n+1}$ and $\left\{g_{n}\right\} \longrightarrow g^{*} \in \overline{B_{d_{l}}\left(g_{0}, \dot{r}\right)}$. Also if $g^{*} \preceq g_{n}$ or $g_{n} \preceq g^{*}$, for all $n \in \mathbb{N} \cup\{0\}$, and inequality (49) holds for all $g, q \in \overline{B_{d_{l}}\left(g_{0}, \dot{r}\right)} \cap$ $\left\{g_{n}\right\} \cup\left\{g^{*}\right\}$. Then $g^{*}$ is a fixed point of $B$ in $\overline{B_{d_{l}}\left(g_{0}, \dot{r}\right)}$.

\section{Data Availability}

The data used to support the findings of this study are included within the article.

\section{Conflicts of Interest}

The authors declare that they have no conflicts of interest.

\section{Authors' Contributions}

Each author equally contributed to this paper and read and approved the final manuscript.

\section{Acknowledgments}

The authors acknowledge with thanks the Deanship of Scientific Research (DSR), King Abdulaziz University, Jeddah, for financial support.

\section{References}

[1] A. Shoaib, A. Hussain, M. Arshad, and A. Azam, "Fixed point results for $\alpha^{\star}-\psi$-Ciric type multivalued mappings on an intersection of a closed ball and a sequence with graph," Journal of Mathematical Analysis, vol. 7, no. 3, pp. 41-50, 2016.

[2] M. Arshad, A. Shoaib, and P. Vetro, "Common Fixed Points of a Pair of Hardy Rogers Type Mappings on a Closed Ball in Ordered Dislocated Metric Spaces," journal of function spaces and applications, vol. 2013, Article ID 638181, 9 pages, 2013.

[3] M. Arshad, A. Shoaib, M. Abbas, and A. Azam, "Fixed points of a pair of Kannan type mappings on a closed ball in ordered partial metric spaces," Miskolc Mathematical Notes. A Publication of the University of Miskolc, vol. 14, no. 3, pp. 769784, 2013.

[4] M. Arshad, A. Azam, M. Abbas, and A. Shoaib, "Fixed points results of dominated mappings on a closed ball in ordered partial metric spaces without continuity," U.P.B. Scientific Bulletin. Series A. Applied Mathematics and Physics, vol. 76, no. 2, pp.123134, 2014

[5] I. Beg, M. Arshad, and A. Shoaib, "Fixed point on a closed ball in ordered dislocated quasi metric spaces," Fixed Point Theory. An International Journal on Fixed Point Theory, Computation and Applications, vol. 16, no. 2, pp. 195-206, 2015.

[6] A. Shoaib, M. Arshad, T. Rasham, and M. Abbas, "Unique fixed point results on closed ball for dislocated quasi $G$-metric spaces," Transactions of A. Razmadze Mathematical Institute, vol. 171, no. 2, pp. 221-230, 2017.

[7] T. Rasham, A. Shoaib, M. Arshad, and S. U. Khan, "Fixed point results for a pair of multivalued mappings on closed ball for new rational type contraction in dislocated metric space," Journal of Inequalities and Special Functions, vol. 8, no. 2, pp. 74-85, 2017.

[8] T. Rasham, A. Shoaib, M. Arshad, and S. U. Khan, "Fixed point results for a pair of multivalued mappings on closed ball for new rational type contraction in dislocated metric spaces," Turkish Journal of Analysis and Number Theory, vol. 5, pp. 86-92, 2017.

[9] T. Rasham, A. Shoaib, B. S. Alamri, and M. Arshad, "Multivalued fixed point results for new generalized F-Dominted contractive mappings on dislocated metric space with application," Journal of Function Spaces, 2018.

[10] T. Rasham, A. Shoaib, N. Hussain, M. Arshad, and S. U. Khan, "Common fixed point results for new Ciric-type rational multivalued $F$-contraction with an application," Journal of Fixed Point Theory and Applications, vol. 20, no. 1, 2018.

[11] T. Rasham, A. Shoaib, C. Park, and M. Arshad, "Fixed point results for a pair of multi dominated mappings on a smallest 
subset in $K$-sequentially dislocated quasi metric space with an application," Journal of Computational Analysis and Applications, vol. 25, no. 5, pp. 975-986, 2018.

[12] T. Rasham, A. Shoaib, M. Arshad, and S. U. Khan, "Fixed point result for new rational type contraction on closed ball for multivalued mapping," Italian Journal of Pure and Applied Mathematics, no. 38, pp. 551-560, 2017.

[13] M. Boriceanu, "Fixed point theory for multivalued generalized contraction on a set with two $b$-metrics," Studia Universitatis Babeş-Bolyai-Series Mathematica, vol. 54, no. 3, pp. 1-14, 2009.

[14] H. Aydi, M.-F. Bota, E. Karapinar, and S. Mitrović, "A fixed point theorem for set-valued quasi-contractions in b-metric spaces," Fixed Point Theory and Applications, vol. 2012, p. 88, 2012.

[15] N. Hussain, J. R. Roshan, V. Parvaneh, and M. Abbas, "Common fixed point results for weak contractive mappings in ordered $b$ dislocated metric spaces with applications," Journal of Inequalities and Applications, pp. 1-21, 2013.

[16] J. Nadler, "Multi-valued contraction mappings," Pacific Journal of Mathematics, vol. 30, pp. 475-488, 1969.

[17] A. Azam and M. Arshad, "Fixed points of a sequence of locally contractive multivalued maps," Computers \& Mathematics with Applications. An International Journal, vol. 57, no. 1, pp. 96-100, 2009.

[18] A. A. Abdou, "Common fixed point results for multi-valued mappings with some examples," The Journal of Nonlinear Science and its Applications, vol. 9, no. 3, pp. 787-798, 2016.

[19] M.-F. Bota, C. Chifu, and E. Karapinar, "Fixed point theorems for generalized $(\alpha *-\psi)$-Ćirić-type contractive multivalued operators in b-metric spaces," Journal of Nonlinear Sciences and Applications, vol. 9, no. 3, pp. 1165-1177, 2016.

[20] M. S. Khan, "Common fixed point theorems for multivalued mappings," Pacific Journal of Mathematics, vol. 95, no. 2, pp. 337347, 1981.

[21] J. H. Asl, S. Rezapour, and N. Shahzad, "On fixed points of $\alpha-\psi$-contractive multifunctions," Fixed Point Theory and Applications, vol. 2012, p. 212, 2012.

[22] M. U. Ali, T. Kamran, and E. Karapinar, "Further discussion on modified multivalued $\alpha^{\star}$ - $\psi$-contractive type mapping," Filomat, vol. 29, no. 8, pp. 1893-1900, 2015.

[23] N. Hussain, J. Ahmad, and A. Azam, "Generalized fixed point theorems for multi-valued $\alpha$ - $\psi$-contractive mappings," Journal of Inequalities and Applications, vol. 2014, p. 348, 2014.

[24] L. B. Ćirić, "A generalization of Banach's contraction principle," Proceedings of the American Mathematical Society, vol. 45, pp. 267-273, 1974.

[25] S. U. Khan, M. Arshad, T. Rasham, and A. Shoaib, "Some new common fixed points of generalized rational contractive mappings in dislocated metric spaces with application," Honam Mathematical Journal, vol. 39, no. 2, pp. 161-174, 2017.

[26] T. Senapati and L. K. Dey, "Common Fixed Point Theorems for Multivalued $\beta^{\star}$ - $\psi$-Contractive Mappings," Thai Journal of Mathematics, vol. 15, no. 3, pp. 747-759, 2017.

[27] J. Jachymski, "The contraction principle for mappings on a metric space with a graph," Proceedings of the American Mathematical Society, vol. 136, no. 4, pp. 1359-1373, 2008.

[28] N. Hussain, S. Al-Mezel, and P. Salimi, "Fixed points for $\psi$ graphic contractions with application to integral equations," Abstract and Applied Analysis, vol. 2013, Article ID 575869, 11 pages, 2013.

[29] F. Bojor, "Fixed point theorems for Reich type contractions on metric spaces with a graph," Nonlinear Analysis: Theory, Methods \& Applications, vol. 75, no. 9, pp. 3895-3901, 2012.
[30] J. Tiammee and S. Suantai, "Coincidence point theorems for graph-preserving multi-valued mappings," Fixed Point Theory and Applications, vol. 2014, p. 70, 2014. 


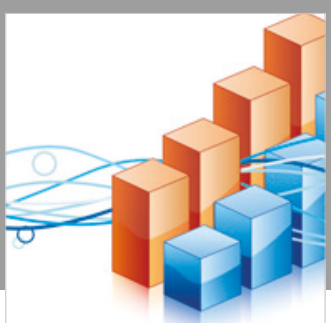

Advances in

Operations Research

\section{-n-m}
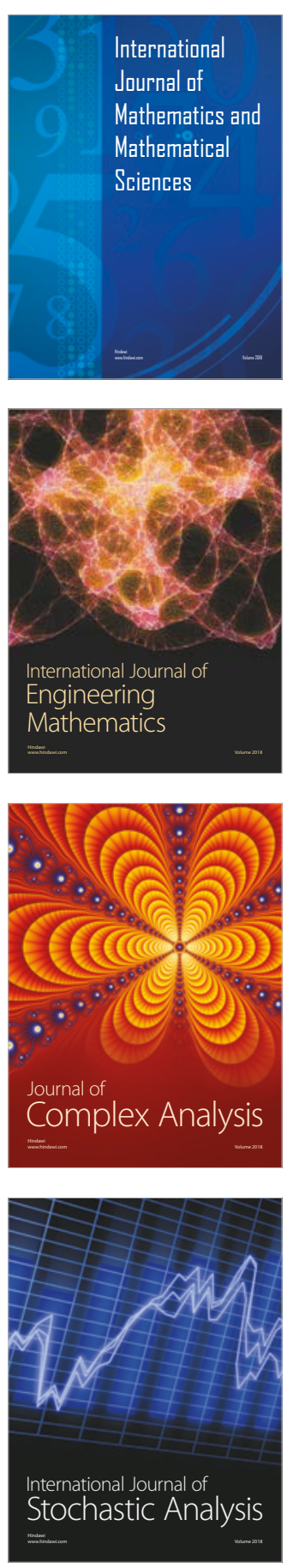
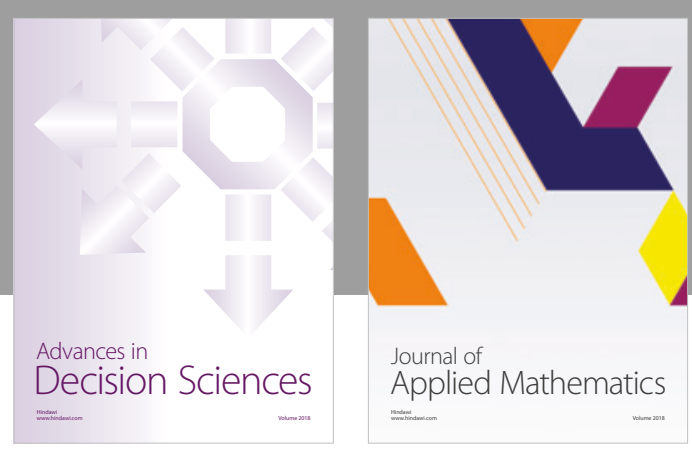

Journal of

Applied Mathematics
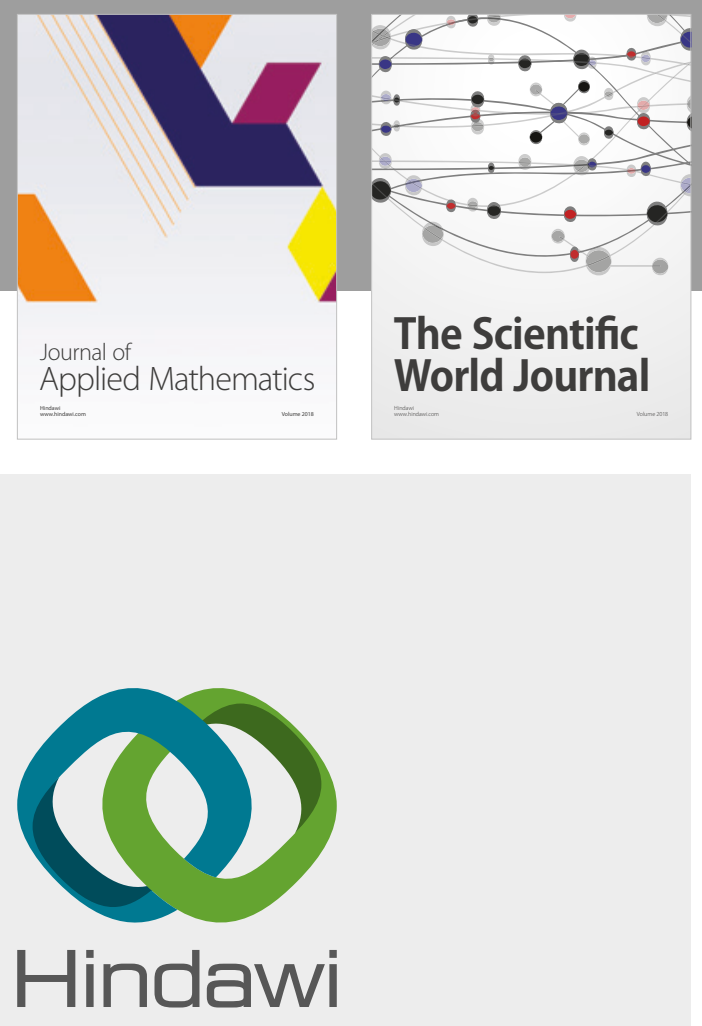

Submit your manuscripts at

www.hindawi.com

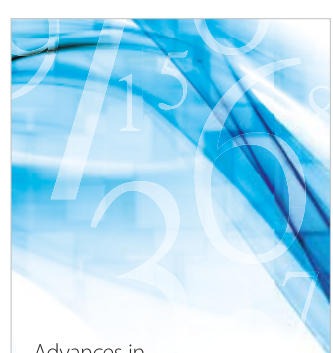

Advances in
Numerical Analysis
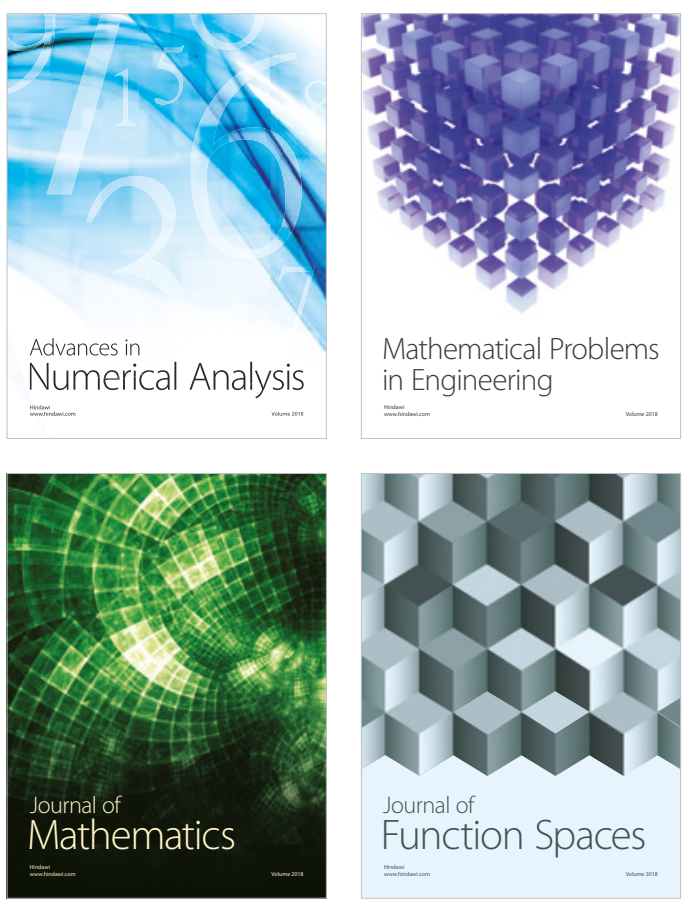

Mathematical Problems in Engineering

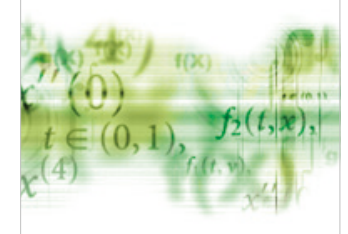

International Journal of

Differential Equations

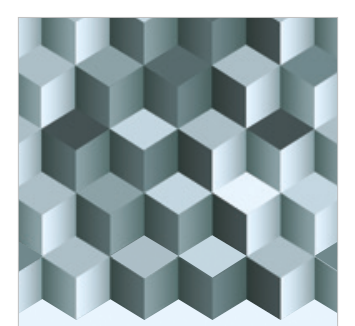

Journal of

Function Spaces

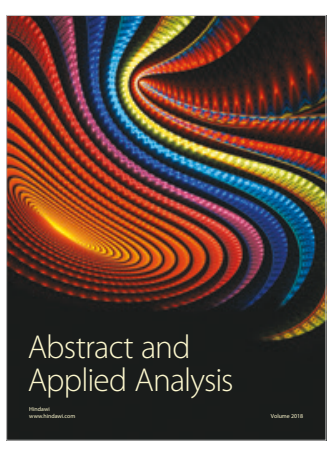

The Scientific

World Journal

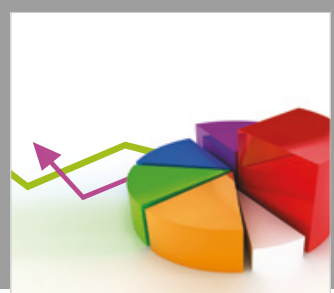

Journal of

Probability and Statistics
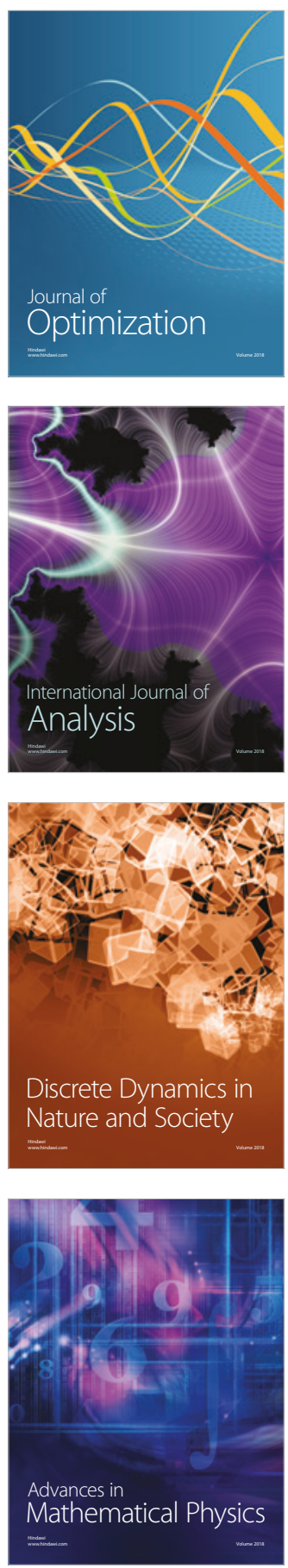